\title{
Utilization of public health centres in Portugal: effect of time costs and other determinants. Finite mixture models applied to truncated samples
}

\author{
Óscar D. Lourenço* and Pedro L. Ferreira \\ School of Economics, University of Coimbra, Coimbra, Portugal
}

\section{Summary}

The impact of time costs on the utilization of medical care has been a subject of theoretical and empirical research since the early 1970s.

The main goal of this paper is to show the effect of time costs on the number of visits to general practitioners (GP) in Portuguese public health centres. We measured the elasticity of primary health care utilization relative to the total time spent in the health centre and relative to travel time. We also provided evidence regarding the impact of an appointment delay on the utilization of public GP services.

Our data resulted from the application of an endogenous sampling scheme, resulting in a truncated-at-zero data set. To model our dependent variable, number of visits, and accounting for the truncated nature of the data we used a finite mixture model specification.

The data were obtained from the most recent implementation in Portugal of the 2003/2004 Europep Survey.

The two-component negative binomial II finite mixture model led to the identification of two different latent classes of health centre users: a low-users class that comprises $88 \%$ of patients with an estimated utilization mean of 4.3 GP visits per year and a frequent-users class with an estimated utilization mean of 11.1 visits for the remaining $12 \%$ of the population.

We failed to find any statistically significant elasticity of time cost utilization, when this variable is measured as the total time spent in the health centre. Regarding the effect of an appointment delay on health centre utilization we concluded that individuals respond to this variable by lowering the number of GP visits. This last finding may have policy implications, which will be discussed at the end of the paper. Copyright (C) 2005 John Wiley \& Sons, Ltd.

Keywords time costs; public primary health care utilization; finite mixture model; truncated sample

\section{Introduction}

This paper aims at assessing the effect of time costs on the demand for general practitioners (GP) in Portuguese public health centres. Despite the theoretical and empirical interest in this issue [1-15], only a few authors in Portugal have tested the impact of time costs on the medical care utilization, mainly on hospital care [14]. This paper provides evidence on this matter describing the results of an empirical analysis of the responses of GP visits to time costs in health centres.

The literature on demand response to time costs usually attaches two meanings to this notion. On the one hand, time cost means the time spent in a waiting list that is, the time waiting for an

*Correspondence to: School of Economics, University of Coimbra, Av. Dias da Silva, 165, 3004-512 Coimbra, Portugal.

E-mail: osl@fe.uc.pt 
appointment with the physician. This sort of time cost is common in health systems characterized by providing free care at the point of delivery [12], which is the case in Portugal. In this paper, this kind of time cost is referred to as 'appointment delay' [6]. On the other hand, time cost may also denote the total time spent by the patient in seeing a doctor, which includes travel time and time spent in the physician office. In this paper, this second variable is referred to as 'physical waiting' [13], encompassing travel time and total time spent in the health centre. Both time cost categories appointment delay and physical waiting - obviously represent a cost to the patient, though intrinsically different. Becker [16], applying a general consumption model, was the first to emphasize time consumed as a cost to the individual. Later, Grossman [17] and Acton [1], among others, applied Becker's ideas to the consumption of medical care and underlined that time costs are a part of the price the patient has to pay to use health care.

In the Portuguese Health System, the extent of these time-related costs may reach significant levels. Cabral [18] found that more than $54 \%$ of the people had to wait more than two weeks to get an appointment with a GP. Relying on data taken from our data set, the average waiting time to visit a GP is approximately 27 days. Regarding the costs associated with physical waiting, the average travel time to the health centre is found to be about $20 \mathrm{~min}$. However, regarding the time spent in the health centre, we found an average of $2 \mathrm{~h}$. Additionally, roughly $17 \%$ of the patients spent more than $3 \mathrm{~h}$ in the $\mathrm{HC}$ and approximately $45 \%$ of the patients reported being unsatisfied with the time spent waiting. Notwithstanding the importance of these time costs to access to a GP, nothing is known about its impact on the utilization of public health centres.

In spite of the existence of a large amount of literature on the effects of time costs on medical care utilization, it is of limited applicability in inferring their effects on the GP utilization in Portugal. Most of the literature on the effects of an appointment delay refers to the hospital setting, mainly for elective surgery [7-12], thus, of little relevance to public primary health care utilization. In addition, the health reasons that drive the demand for primary health care are totally different from those related to hospital care, therefore precluding the direct extrapolation of the results obtained in these studies to the
Portuguese GP demand. Concerning the studies dealing with the effects of physical waiting in the utilization of medical services $[1-6,19,20]$ it is also difficult to extrapolate their findings to the demand of GP services. Besides being a little outdated, almost all these studies analyse medical care categories different from those examined in this paper, and more important, in health systems with different organizations.

Summarizing, the major goal of this paper is to study the effect of time costs on the number of visits to the GP in health centres. To attain that goal we measured the elasticity of GP utilization relative to the total time spent in the health centre and relative to the travel time. We also provide evidence about the impact of an appointment delay on the GP demand. In addition, given the use of regression models, this study also allows the assessment of the importance of other determinants of health centre utilization, e.g. age, gender, education, labour activity status, health status and of the effect of other relevant variables which are potential determinants of utilization.

The present study could be important on several grounds. First, because by providing empirical evidence on this subject, we contribute to the literature on the effect of time costs on the utilization of medical care. Second, it will shed extra light on a subject about which there is little empirical evidence for Portugal [14]. The uncovering of such empirical evidence may be important for policy making in particular, because it may help to understand the effect of policies aiming at increasing the access to health centres, and at the decreasing physical waiting times. Learning about the impact of other variables on the utilization of primary care may help health centre managers and policy makers to understand why patients with comparable health conditions vary in the use of public GPs. This may be important to a better understanding of the behaviour of users and may provide insights about organizational changes which could improve the effectiveness of this type of public providers, especially in countries with a gatekeeper system implemented.

A last contribution of this paper is methodological. We apply count data finite mixture models (FMMs) to a data set generated by applying an endogenous sampling scheme. As noticed by Santos-Silva [21], the application of mixture models to data collected with endogenous sampling, presents several methodological difficulties that deserve discussion. 
In this paper, health centre utilization is measured using the number of visits to the GP in the 12 months prior to the application of the survey. The data for the empirical analysis were taken from the 2003/2004 Europep Survey [22], a health centre's users survey that is representative of the users of all public health centres, and administered in a non-regular basis.

The remainder of this paper contains six sections. In Section 2 we present some information regarding the organization of the Portuguese Health System, emphasizing the public primary health care sector, while in Section 3 we describe the data collection methods, specify and describe the variables that are used in our model, showing also some descriptive statistics. Section 4 discusses the empirical methods used to analyse the data. In Section 5, the empirical results are presented and in the next section, 6, the results are discussed. Finally, Section 7, the conclusion, finalizes the paper.

\section{Background of the Portuguese Health System}

In Portugal, three co-existing systems provide health care coverage to the population: (1) the National Health Service (NHS) financing the large majority of the medical care; (2) public and private occupational health insurance coverage, referred to as health subsystems [23]; (3) voluntary health insurance. Citizens with occupational insurance represent $20-25 \%$ of the population, mainly the well-off individuals. Therefore, the Portuguese Health System is financed by a mix of public and private sources, with high level of private expenditure, when compared to health systems based on a NHS [24].

Looking particularly at the public primary health care, the delivery of health care is provided through a large network of roughly 360 public health centres employing a total of 30000 professionals, $25 \%$ of them GPs and $20 \%$ nurses, both paid on a salaried basis. The care provided by these health centres includes general medical care for adults, children's care, women's health, family planning, prenatal and perinatal care, as well as first aid. They also perform bureaucratic tasks such as certification of incapacity to work and capacity for some jobs. Home visits and preventive services are also provided by the health centres [23]. Each GP is responsible for a given number of patients, ranging from 1000 to 2000 patients. The visit to a public GP has a low monetary cost to the patient (a regular visit costs $2 €$ and an emergency visit costs 2,70€) and some patients - e.g. children under 12 , pregnant women, some chronic patients, unemployed individuals and individuals with earnings under the minimum national wage, etc. - are exempt from this payment [25].

GPs are expected to act as gatekeepers to the system. Therefore, unless admitted from the emergency care unit, patients should have a prior visit to a GP to get the appropriate referral to a public hospital specialist. Moreover, patients must be registered with a GP in the health centre and can freely choose him/her within a geographical area, as long as the physician has a vacancy in his/ her patient list. It is expected that individuals demand public GP services in the health centre where they are registered. There are no limits on the number of visits that an individual can make to the GP. Even citizens without an assigned GP may go to a health centre for health care and receive the same treatment as those on the GP's list. Furthermore, about $30 \%$ of patients who go to a health centre without previously schedule appointment see a GP in the same day [22].

On the other hand, the visit to a private GP is, in general, always an alternative, requiring however the full payment of the visit for those individuals without voluntary health insurance or subsystem coverage. Subsystems beneficiaries and voluntary health insurance holders have the same opportunities as NHS beneficiaries to use GP services in the health centre [25], nonetheless, they rarely use public primary medical care services. From data taken from the National Health Survey 1998/99, we found that, in 3 months, only $9 \%$ of the individuals that made at least one visit to the health centre were enrolled in a subsystem or voluntary health insurance plan. These figures suggest that the users of public GPs are those individuals covered only by the NHS, while the subsystems enrollees and voluntary health insurance holders form a group of users who demand almost all their ambulatory care from the private sector.

As a result of an uneven distribution of medical resources across the country, individuals living in poorer and isolated geographical areas have less access to health care $[23,26]$. More importantly, as private provision is greatly concentrated in the 
regions where NHS supply is more extensive [27], people living in geographically isolated areas also lack private alternatives [28]. This feature of the Portuguese Health System may be important to understand the empirical results of this paper.

Along with providing health insurance through a NHS, the Portuguese State also runs a Social Security System that provides a network to help the citizens in financially difficult situations, for instance in the occurrence of an illness that prevents the individual from work, and the resulting loss of income. So, Portuguese workers are protected by sick leave policies, designed to provide salary continuity in the event of illness, against loss of income resulting from illness. However, this policy does not guarantee the payment of the full salary to the sick worker as the total payment is indexed to the number of sick days. For instance, if the illness prevents the individual from working less than 30 days, the social security only reimburse $55 \%$ of the individuals' salary [29], and this payment is made only after the fourth day of illness. This means that in the first 4 days of sick leave the worker loses income. More importantly, visits to the doctor also mean the loss of income as the time needed to see the physician is not reimbursed by the Social Security System.

\section{Data and variables}

As mentioned in the introduction, the data for the empirical analysis were taken from the 2003/2004 Europep Survey [22]. This survey is representative of the users of public health centres, is administered in a non-regular basis and was originally designed to obtain satisfaction scores from the health centre's users. It was based on the Europep questionnaire, created by an international task force on patient evaluation of general practice care $[30,31]$ and financed by the European Union.

The process used to select the individuals was as follows: First, we obtained a list of patients who visited all health centres during the last 3 months of 2003 and from that list we deleted multiple records related to the same individuals. Second, from this list with 1575061 patients, a random sample - proportional to the distribution of age and gender within each health centre - of approximately 67000 patients was taken, and questionnaires were directly mailed to user's residencies. The questionnaire was filled in by patients and sent back in a prepaid envelope, so anonymity was guaranteed. We have received 11166 questionnaires, a rate of response of approximately $17 \%$. After deletion of the records with missing values, on at least one variable of interest, and respondents aged below 18, our final workable sample size was 6791 individuals.

The resulting total sample is representative by age and gender of the actual users. In fact, $\chi^{2}$ tests with both variables within each health district showed non-significance. After removing about 4000 cases due to missing values, as mentioned above, we compared our data with data taken from the National Health Survey 1998/99. Using the variables age, gender, education, occupation and some chronic health conditions we did not find significant differences. Moreover, the estimation of a probit model with a dummy dependent variable that equals 1 if the record enters in the regression and 0 otherwise, and number of visits as independent variable, showed that this last variable is not statistically significant. Similar probit models with different independent variables led us to similar conclusions. Therefore, we may conclude that the deletion did not introduce any bias in our data set.

Due to the sampling scheme - we selected a sample from the population of individuals that visited the doctor in a given period - we did not observe the entire distribution of the dependent variable, in particular individuals with zero visits. Therefore, the dependent variable is truncated-atzero. Table 1 shows the empirical distribution of the dependent variable.

Results showed that $57 \%$ of individuals visited a GP less than five times in a year. Actually, the maximum number of visits taken was 48 , the average number of visits was 5.5 visits with a standard deviation of 5.3 and a median of 4 visits.

The covariates used in this paper were those generally used in other studies on the determinants of health care utilization, being their selection based on theoretical models developed by other authors $[1,2,17,32]$. Table 2 shows the definitions and summary statistics of the covariates, which are assumed to be determinants of the health centre utilization.

We grouped the covariates into six categories, encompassing socioeconomic, health status, time costs, consultation, supply and interaction variables. Next, follows a brief description of each group of variables. 
Table 1. Empirical frequency distribution of the number of visits to the GP

\begin{tabular}{rrrrrc}
\hline Count & Frequency & Relative frequency & Count & Frequency & Relative frequency \\
\hline 1 & 1065 & 15.68 & 20 & 106 & 1.56 \\
2 & 986 & 14.52 & 21 & 2 & 0.03 \\
3 & 906 & 13.34 & 22 & 5 & 0.07 \\
4 & 909 & 13.39 & 24 & 15 & 0.22 \\
5 & 511 & 7.52 & 25 & 10 & 0.15 \\
6 & 653 & 9.62 & 26 & 7 & 0.03 \\
7 & 178 & 2.62 & 27 & 2 & 0.04 \\
8 & 303 & 4.46 & 28 & 3 & 0.01 \\
9 & 76 & 1.12 & 29 & 1 & 0.54 \\
10 & 403 & 5.93 & 30 & 37 & 0.04 \\
11 & 23 & 0.34 & 32 & 3 & 0.04 \\
12 & 368 & 5.42 & 35 & 4 & 0.01 \\
13 & 16 & 0.24 & 36 & 3 & 0.16 \\
14 & 32 & 0.47 & 38 & 1 & 0.03 \\
15 & 91 & 1.34 & 40 & 11 & 0.12 \\
16 & 18 & 0.27 & 41 & 2 & 0.01 \\
17 & 4 & 0.06 & 42 & 8 & 0.03 \\
18 & 21 & 0.31 & 44 & 1 & 0.01 \\
19 & 4 & 0.06 & 47 & 2 & \\
\hline
\end{tabular}

In the first group we included the variables 'male', 'age', 'education' and a variable to capture the opportunity cost of time, 'non-active'. Two additional variables were included and measured at county level, the purchasing power of the county [33] and the percentage of urban population in each county. These variables were created with data provided by the National Statistical Institute (INE) and were merged in our data set.

To measure health status we used self-assessed health (SAH) and a set of dummy variables indicating the existence of a chronic condition. We also include the dummy variable 'sick' that equals one if the last visit to the doctor was requested due to a health shock.

The survey supplied information regarding time costs in the last visit. Three variables were selected. Two of them, representatives of physical waiting ('travel_time and 'hc_time') represent the time the individual spend to visit the GP. A third variable ('app_delay') represents the cost associated with waiting in a list and equals 1 if the individual suffered an appointment delay in the last visit and 0 otherwise. We opted for using this dummy instead of the integer variable representing the number of days waiting for the visit, because a large number of individuals failed to respond, creating many missing values.
The supply of health services was also included and measured as the number of health centre GPs per 1000 inhabitants at the county level. The values needed to compute this variable, population and number of GPs, were obtained in INE.

We also included in the regression three additional variables that may have an effect on health centre utilization. The variable 'not_enought_time', is a dummy considered as being related to the quality of care dimension (equals 1 if the individual is not satisfied with the consultation time and 0 otherwise). The remaining two variables in this section include a variable encompassing the person who instigated the visit and another indicating whether the individual travelled to the health centre on foot. This last variable was included to control for the effect of transportation mean in the travel time.

A set of interaction variables were also created and inserted in the regression model (see Table 2).

Multicollinearity problems may arise in utilization related models using a high number of covariates. Through visual inspection of the independent variable correlation matrix we found that nearly all correlations were less than 0.3 , with the majority having values less than 0.1 . However, the variable 'density of physicians' had a correlation of 0.6 and 0.7 , respectively, with 'logarithm of 


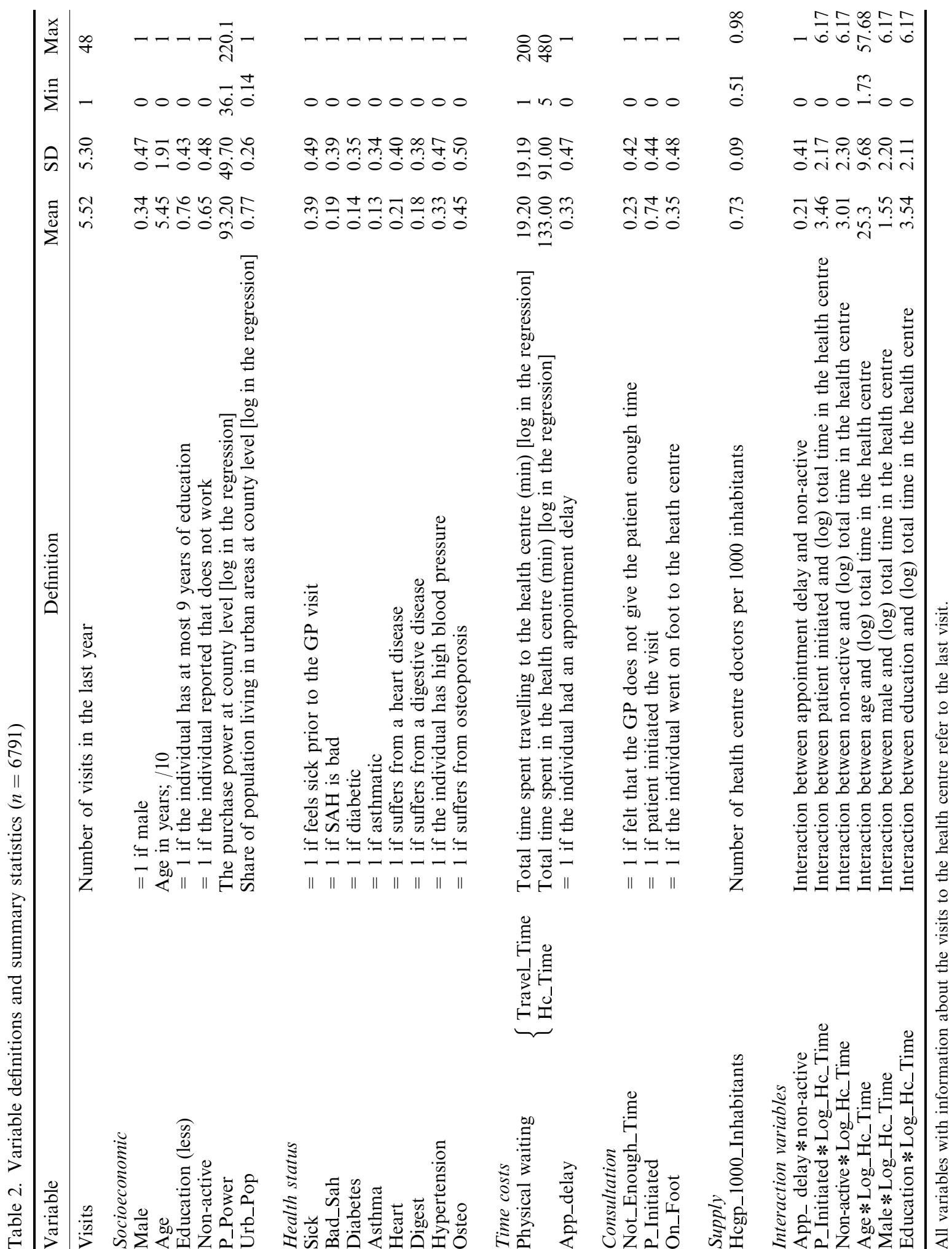


purchasing power' and 'logarithm of urban population'. We proceeded as Oliveira [14] and eliminated the covariate that represented the density of physicians. After eliminating this variable the problem of high correlations was overcome.

\section{Econometric specification}

In this section we discuss the specification of an econometric model acknowledging the fact that the dependent variable - the number of visits to a public GP - always takes integer and positive values, and unobserved heterogeneity is supposed to exist. Hence, the specification must incorporate this unobserved heterogeneity. Santos-Silva [21] has shown that the specification of mixture models, continuous or finite, applied to data collected with endogenous sampling merits special attention.

The natural starting point to analyse count data is through a Poisson regression model which may be restrictive in several ways [34]. To overcome this constraint, alternative count data models have been proposed, (e.g. two-part models - TPM), usually referred to as a hurdle model when applied to count data. Hurdle models have been considered as cornerstone in the modelling of medical care services [35].

Hurdle models assume that the mechanism governing individuals with zero counts (non-users) is different from the statistical process ruling the positive observations (users). They also assume that the full population is a mixture of two subpopulations, the subpopulation of users and the subpopulation of non-users of health care, each being described by a different statistical process $[21,36]$. In the context of the Portuguese primary health care system, these assumptions are suitable as there is a segment of the population that seldom use a health centre. Those are individuals covered by a subsystem or by a voluntary health insurance, usually the better-off, which tend to prefer the private sector.

To estimate hurdle models a random sample of the entire population of interest should be available, though this is not our case. As it was previously described our sampling scheme only allows us to observe the population of users, therefore the full hurdle model cannot be estimated. Hence, due to sampling design specifications we are constrained to estimate only the second part of the hurdle, the part that describes the distribution of the users.

Models allowing for truncation have been the focus of much research, and several papers have analysed data with this characteristic [37-39], though applications to medical care data are quite uncommon. Considering $f\left(y_{i} \mid x_{i}\right)$ the density function of the $i$ th person in the population, the corresponding density in the truncated sample is given by [37-40]

$f\left(y_{i} \mid x_{i}, y_{i}>0\right)=f_{s}\left(y_{i} \mid x_{i}\right)=\frac{f\left(y_{i} \mid x_{i}\right)}{1-F\left(0 \mid x_{i}\right)} \quad y_{i}>0$

where $f($.$) is the probability function in the$ population and $F($.$) is the corresponding distribu-$ tion function.

Ignoring unobserved heterogeneity in truncated models leads to inconsistent parameters estimates [21,36-38]. To specify the model to accommodate unobserved heterogeneity, a continuous or finite mixture model specification is required, and instead of specify the distribution of $\left(y_{i} \mid x_{i}\right)$, the specification of the distribution of $\left(y_{i} \mid x_{i}, v_{i}\right)$ is required [36] along with the specification of distributional assumptions regarding the random variable $v_{i}$, which represents the unobserved heterogeneity. If the random variable $v_{i}$ is assumed to be continuous, then a continuous mixture model is specified, conversely, when $v_{i}$ is assumed to be discrete then a semi-parametric finite mixture model (FMM) is specified [35,41]. The finite mixture approach has been used by Deb and Trivedi [35, 42], Deb and Holmes [43], Gerdtham and Trivedi [44], Bago d'Uva [Latent class models for use of primary care: evidence from a British Panel. Health Econ, in press], Atella et al. [45], among others, to study medical care utilization when a random sample selected from the entire population was available.

FMMs - or latent class models as named by Deb and Trivedi [35] and Wedel et al. [46] - offer a number of advantages over the use of continuous models. Deb and Trivedi [35], Cameron and Trivedi [36] and Wedel et al. [46] provide a rather complete list of the advantages. First, Heckman and Singer [41] show that estimates of a finite mixture might provide good numerical approximations even when the distribution of $v_{i}$ is continuous; second, provides a natural and intuitively attractive representation of unobserved heterogeneity in a finite, usually small, number of latent classes, each of which may be regarded as a 'type' or 'group [35]'. Moreover, empirical investigation has shown 
that FMM provide good results $[35,36,42,43]$. Additionally, the potential for identification of latent classes of individuals, based on non-observable characteristics, may be important for interpreting reasons because the impact of some variable may differ across latent classes, and, with little algebra, the impact of this same variable on the population is equally possible to discern. Therefore, there are good reasons for specifications based on FMM applied to data originating from random sampling of the relevant population.

To formulate a FMM let us assume that $v_{i}$ follows a discrete distribution with $c$ support points, $v_{i}^{k}(k=1,2, \ldots, c)$, with probability masses $\pi_{k} \geqslant 0(k=1,2, \ldots, c)$, with $\sum_{k=1}^{c} \pi_{k}=1$. Moreover, let us assume that the distribution of $\left(y_{i} \mid x_{i}\right.$, $\left.v_{i}^{k}\right)$ is $f_{k}\left(y_{i} \mid x_{i}, v_{i}^{k}, \theta_{k}\right), k=1, \ldots, c$, where $\theta_{k}$ is a vector of parameters. Under these conditions, the distribution of $\left(y_{i} \mid x_{i}\right)$, is given by

$f\left(y_{i} \mid x_{i}, \theta\right)=\sum_{k=1}^{c} \pi_{k} f_{k}\left(y_{i} \mid x_{i}, v_{i}^{k}, \theta_{k}\right)$

where $\theta^{\prime}=\left[\theta_{1}, \ldots, \theta_{k}\right]$ represents a vector of parameters. The mixing probabilities $\pi_{k}(k=1, \ldots, c)$ are estimated together with all other model parameters so the estimation procedure also provides an estimate of the mixing distribution [38].

Brannas and Rosenqvist [38] claim that in the truncated context it is even more relevant the utilization of FMM. Santos-Silva [21] showed that when $v_{i}$ is assumed to be distributed in the actual population, then

$f_{s}\left(y_{i} \mid x_{i}, \theta\right)=\sum_{k=1}^{c} \frac{f_{k}\left(y_{i} \mid x_{i}, \theta_{k}\right)}{\left(1-\sum_{k=1}^{c} \pi_{k} F_{k}\left(0 \mid x_{i}, \theta_{k}\right)\right)} \pi_{k}$

Conversely, when $v_{i}$ is assumed to be distributed in the truncated population, then

$f_{s}\left(y_{i} \mid x_{i}, \theta\right)=\sum_{k=1}^{c} \frac{f_{k}\left(y_{i} \mid x_{i}, \theta_{k}\right)}{\left[1-F_{k}\left(0 \mid x_{i}, \theta_{k}\right)\right]} \hat{\pi}_{k}$

This same author also asserts that in the case of hurdle models the truncated population is of interest in itself, therefore it is natural to estimate the model for positives by specifying the unobserved heterogeneity in the population induced by the sampling scheme, thus using specification (4).

In this paper, we estimated models with $c=2$ and 3. Regarding the distribution of the component densities we chose the most usual densities applied to model count data, that is, Poisson, negative binomial I and II $[35,36,42,43]$. We estimate several FMMs which were compared by using Bayesian information criteria (BIC) and consistent Akaike information criteria (CAIC) measures [47].

All models were estimated using STATA 8.0, which solved our unconstrained maximization problems relying on the Broyden-Fletcher-Goldfarb-Shanno algorithm. Robust standard errors of parameter estimates are reported [36].

Due to the exponential mean specification, marginal effects differ between individuals. In this paper, we computed average marginal effects (AME), that is, we computed the marginal effect for each individual and averaged over the sample, a procedure considered conceptually preferable by Cameron and Trivedi [36]. To estimate AME standard errors we used the Delta method [48].

\section{Empirical results}

In this section we report the results of the empirical analysis, beginning with the results of model selection methods used to choose the preferred FMM in terms of the number of classes and the component distributions.

Models with $c=1$ and 2 are nested, so it is possible to use LR tests to choose among them $[36,42]$. Models with $c=1$ were rejected, remaining the comparison of $c=2$ and 3. Table 3 shows the values of BIC and CAIC for various FMM with $c=2$ and 3 , and Poisson, negative binomial I and II component distributions.

Based on the full sample, both BIC and CAIC indicators support the two-component negative binomial II FMM. Moreover, the estimate of $\pi$ is large relative to its estimated standard error, and as argued by Cameron and Trivedi $[36,42]$, this evidence supports the FMM with two latent classes. Here we only report the results from FMM with two classes and negative binomial II as component distribution.

Table 4 presents the sample average of the estimates of the fitted means by latent classes, along with some other summary statistics. Low users, comprise $88 \%$ of the population, have on average, 4.3 visits annually to the public GP, whilst the remaining $12 \%$, the frequent users, seek care 11.2 times a year, almost one visit per month.

Table 5 shows the parameter estimates, AME and $z$-values for the two latent classes FMM. A scan through the table shows that the coefficients 
Table 3. Information Criteria (BIC and CAIC) to select among non-nested models

\begin{tabular}{|c|c|c|c|c|}
\hline & \multicolumn{2}{|c|}{ Two-component finite mixture } & \multicolumn{2}{|c|}{ Three-component finite mixture } \\
\hline & $\mathrm{BIC}$ & CAIC & $\mathrm{BIC}$ & CAIC \\
\hline Poisson & 36751 & 36808 & 35345 & 35431 \\
\hline Negative binomial I & 34967 & 35026 & 35053 & 35142 \\
\hline Negative binomial II & $34921^{*}$ & 34980 & 35115 & 35204 \\
\hline
\end{tabular}

*Preferred model.

Table 4. Distribution of fitted mean values by latent class

\begin{tabular}{lccc}
\hline Statistic & $\begin{array}{c}\text { Class I } \\
\text { Low users }\end{array}$ & $\begin{array}{c}\text { Class II } \\
\text { Frequent users }\end{array}$ & Population \\
\hline Mean & 4.3 & 11.1 & 5.12 \\
Min & 1.3 & 2.6 & 1.9 \\
Max & 15.1 & 33.0 & 15.9 \\
& & & \\
Percentile & & & \\
10 & 2.49 & 6.85 & 3.15 \\
25 & 3.06 & 8.50 & 3.79 \\
50 & 3.98 & 10.66 & 4.80 \\
75 & 5.22 & 13.27 & 6.07 \\
90 & 6.65 & 16.02 & 7.57 \\
\hline
\end{tabular}

in most variables have the expected signs and also that the majority of them are statistically significant, mainly in class I. It is also perceptible that the effects of some covariates, measured by the AME, are different across the latent classes.

The gender effect is negative $(\mathrm{AME}=-0.597)$ among the low users; that is, the infrequent male users tend to use the care provided by health centres less often than the female. The effect of gender in the frequent-users class is also negative, however, without statistical significance. Age did not show any statistical significance in both classes, even with age square in the model. The results also show that less education is a factor that increases the utilization of the health centre. This result is statistically significant and similar between the two classes of users. In the low-users class non-active individuals visit the health centre more often than the active ones. This same variable is not statistically significant in the frequent-users class. The remaining variables in the socioeconomic group, purchasing power and share of urban population have a negative effect in utilization, however, in different latent classes. The elasticity of utilization relative to purchasing power $(-0.218)$ is negative in the frequent-users class, while the elasticity of utilization relative to the share of urban population $(-0.121)$ is statistically significant only in the low-users class.

The covariates representing health status variables, both objective health status variables (chronic disease) and $\mathrm{SAH}$, have positive and statistically significant effects on the low-users class but not on the frequent-users latent class. This means that, in the low-users class, individuals that reported bad SAH and those with chronic conditions use the GP more often. In the frequentusers class only those individuals who suffer from heart problems seeks GP visits more often.

Regarding the effect of the total time spent in the health centre, after computing the elasticity accounting for the interactions, we conclude for each latent class, that this variable is not relevant to explain utilization. In both latent classes, the elasticity of utilization relative to total time spent in the health centre is not statistically significant. We also computed the elasticity for the entire population and we obtained a negative elasticity of -0.078 , however without statistical significance. Additionally, parameters of the interaction variables did not present any statistical significance. On the other hand, the elasticity of the utilization relative to travel time is positive and statistically significant in both classes. Their magnitudes are different across both classes: in the low-users class the effect of travel time is small; conversely, in the class of frequent users an elasticity of 0.125 was found. This means that when travel time increases $10 \%$, the average utilization increase $1.25 \%$. Regarding the effect of the appointment delay variable, we found a statistically significant large negative effect, in both latent classes. This effect is lower $(-0.720)$ in the low-users class compared with the AME obtained (-2.150) for the frequentusers class. To further investigate whether this effect is equal for both active and non-active individuals we computed the interaction effect of appointment delay on the occupation variable. As 
Table 5. Parameter estimates, average marginal effects and $z$-stats for the two-component negative binomial II finite mixture model

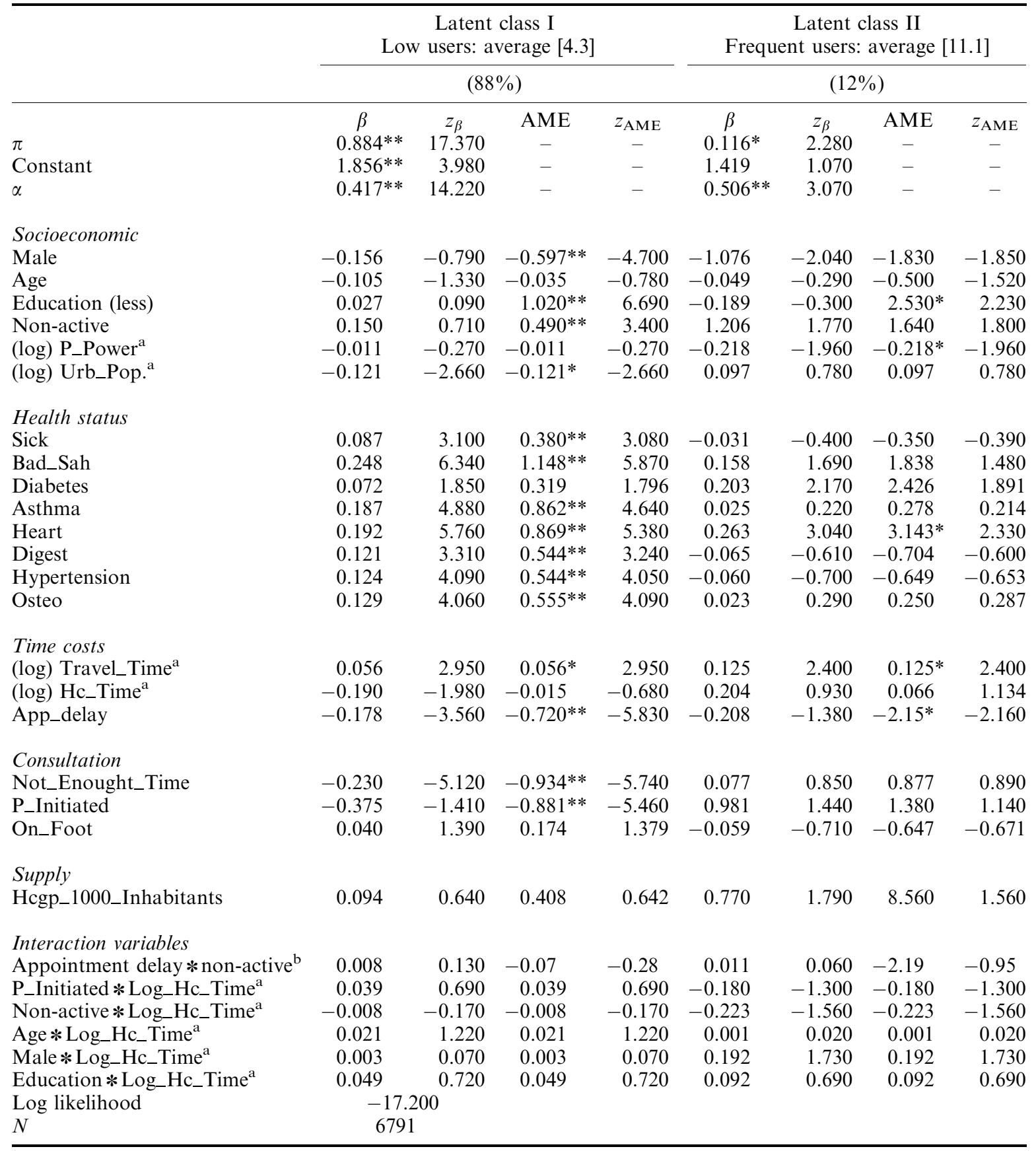

*Statistically significant at $5 \%$ level.

** Statistically significant at $1 \%$ level.

${ }^{\mathrm{a}} \mathrm{AME}$ measure elasticities.

${ }^{\mathrm{b}} \mathrm{AME}$ measure the interaction effect between the two variables.

Marginal effects calculated for each individual and averaged over the sample. AME $z$-stats computed by using the Delta method. AME for dummy variables are discrete changes from 1 to 0 . 
shown, the interaction effects in both latent classes are non-significant.

Within the group of variables called 'consultation' we only highlight the perception of adequacy of the time given by the doctor for the consultation ('not_enought_time'). The AME of this variable in the low-users group is negative and statistically significant.

Finally, the parameter estimate for public GP supply did not show any effect on the utilization, in both latent classes.

\section{Discussion}

In this section we will discuss the most relevant results of the current study.

The main purpose of this paper was to model the utilization of health centres in Portugal and to detect its major determinants. Among several independent variables, special attention was paid to the impact of some time-cost variables, namely the total waiting time in the health centre, the time spent by patients travelling to it and the existence of an appointment delay. The utilization was measured by the number of visits to the health centre in the 12 months prior to the filling of the questionnaire, generating a count variable. The corresponding sample was collected among the users of each health centre during a given time period. Due to this sampling scheme the dependent variable is truncated at zero and we used modified finite mixture models to model it. Our modelling framework led us to the identification of two different latent classes of health centre users: a low-users class that comprises $88 \%$ of patients with an estimated utilization mean of 4.3 visits in the course of an year, and a frequent-users class with an estimated utilization mean of 11.1 visits for the remaining $12 \%$ of the population. The rationale used to select the covariates and to interpret their effect on the utilization was based on theoretical models developed by other authors $[1,2,17,32]$.

The expected effect of a variable in the utilization of public GPs is the composition of two indistinguishable effects. We must consider the impact of the variable on the total medical care utilization, as well as, its effect on the substitution of public GPs for private physicians or emergency care units.

Regarding the number of latent classes our results are similar to those obtained in other studies $[35,42-$
45] (Bago d'Uva, in press), which have found that FMM with two classes fitted the counts well enough. It is interesting to note that models with $c=3$ are preferred when the component distributions are Poisson; in contrast, models with $c=2$ are the preferred for negative binomial distributions. The justification is that when negative binomial distributions are used, unobserved heterogeneity is accommodated in two ways: through the mixing distribution and through the component densities which, being negative binomial, can accommodate unobserved heterogeneity present in each latent class. When the Poisson is used as component distribution, the unobserved heterogeneity is accommodated only through the mixing distribution, which now needs one extra mass point to satisfactorily represent the unobserved heterogeneity.

\section{Time costs}

The time spent travelling to and from the health centre and the total time spent in the health centre, are considered a cost to the individual because this time could have been used to earn income, for leisure or some other alternative utilization. Acton [1] shows that the necessary assumptions that make money function as a price in determining the demand for health care are sufficient to make time function as a price. Furthermore, the impact of this kind of time costs is stronger when the monetary cost is low relative to the time cost $[1,2]$. We then anticipate that travel time and time spent at the health centre are factors which, on average, decrease utilization. Both effects, impact on the total demand and on the substitution effect, may be at work here.

The appointment delay is seen as the number of days between the appointment and the consultation. As Lindsay and Feigenbaum [7] put it, the time spent on a waiting list has a zero cost, however, the value of a good is lower if received later than sooner. Propper [12] disagreed with this vision and argued that for medical care this kind of waiting entails a cost to the patient. Therefore, the longer the appointment delay the fewer visits are made to the health centre. This is because in the event of a long waiting, the relative cost of seeing a private physician is becoming lower and accordingly people are more willing to substitute public care for private care. This may be true even in Portugal with a Social Security System providing sick leave policies. 
As shown by several authors $[1,2,4,11,19]$, both categories of time-costs are a factor that shifts the demand for medical care. This assertion is particularly true in health systems where the money price is relatively unimportant, and times costs emerge naturally as a device to allocate resources [1,12]. Acton [1] argued that, in a setting where the monetary price of health care is null or near null, a mechanism involving time is quite likely to assume the role of demand shifter, since consumption of health care usually entails a cost in travel time and waiting time.

Our empirical results regarding physical waiting contradict the rationale that total time spent at the health centre and travel time would be a relevant cost and, consequently, a factor that would decrease utilization. Our estimates have revealed that utilization is highly inelastic to the total time spent at the health centre. The explanation for this result may reside on the characteristics of the population of health centre users. They are the worse off, with less economic power, and thus with less capacity to seek care outside the public system. Coffey [3] found similar results on ambulatory female medical care services and other authors have come to similar conclusions $[49,50]$. Concerning the positive travel time elasticity in both latent classes, this result also contradicts other empirical findings $[1,2,5,51]$, although some other studies report that the utilization does not respond to travel time [52]. It should be noted, however, that most of these studies use discrete choice models to explain the choice of the medical provider and we used a total utilization variable. Our results may be the consequence of the health centres distribution across the country, and especially the uneven distribution of private medical facilities throughout the country, highly concentrated in urban areas [28]. In fact, in the Portuguese context, people living far from the health centre generally live in rural areas where private and emergence care alternatives are slim. Therefore, the ones with higher travel times also have less possibility to substitute primary care. Contrary to the effect of time costs related to physical waiting, the appointment delay parameter presents, in both latent classes, the anticipated negative sign and patients see the time spent in a list as a cost. Cauley [6] found similar results regarding this variable. This result shows that for most patients there is a selfestablished delay, based on which patients leave the doctor and initiate self-treatment, or seek for alternatives in the private sector or in emergency care units. Barros and Olivella [15] state that this delay depends on the individual health status. In our study frequent-users are more responsive to a delay than less frequent users, showing that the waiting in a list represents a heavy burden.

\section{Socioeconomic variables}

The variable gender may capture biological differences, as well as differences in life styles or attitudes towards risk [53]. Utilization related studies generally have found that male individuals seek less ambulatory care $[1,4,6,35]$. Furthermore, Oliveira and Bevan [28], reporting to the hospital utilization in Portugal, found that male users present lower levels of utilization but a more complex case-mix. Given our available empirical evidence this variable showed a negative effect on utilization. This result may be due to a more preventive behaviour of female individuals in what concerns health issues. Women with a more preventive attitude use the primary care more often.

Regarding the impact of age in utilization, Grossman predicts that the depreciation rate of health capital increases with age, and if the price elasticity of demand for health is less than one then the demand for health services increases as the individual ages [17]. On the other hand, Acton [1] also found that age is a factor that raises the utilization of public care; therefore we expected that age increases the utilization of public GPs. However, our results did not show any statistically significant parameter regarding this variable.

Education increases the productive efficiency of health inputs, meaning that given the occurrence of a disease, individuals with a higher level of education are more likely to identify early signs of illness, seek out medical care, and effectively face the unfavourable health problem [17]. Therefore, education has a negative effect on the total demand for medical care. Regarding the effect of education on the utilization of private care, Acton [1] reported that education is a factor that decreases the utilization of public physicians and increases the utilization of private doctors. So, education may be a factor that induces the substitution of public by private care, and so, this effect will also decrease HC utilization. Because education is measured as a dummy 
variable that equals one if the individual has at most 9 years of schooling, the estimated positive parameter of education go in line with those mentioned above.

Another factor accounted for in the model is occupation, represented by the variable nonactive. Because inactive individuals may attach lower opportunity cost to their time relatively to the active ones, we foresaw a positive effect on the GP utilization. Moreover, individuals with a job may be more willing to substitute public for private care because generally private care may be, for instance, scheduled to the end of the day, therefore, imposing a lower opportunity cost in terms of loss of income. This was confirmed in our study, especially on the low-users class. The nonstatistically significant results for the frequentusers class may be de to the fact that they are mainly individuals with a stringer need for continued care and so, more associated to preprogrammed plan of visits.

As presented in the previous section, the urban population variable, representing the percentage of urban population who lives in each county, has a negative elasticity in the low-users class. In our view the contribution of the substitution effect dominates over the effect of this variable on total demand. This result may be caused by the large geographic inequalities in the distribution of private medical care facilities, which tend to be concentrated in urban and coastal areas [28]. Therefore, for individuals living in urban areas, the time costs for seeing a private physician are lower than the time costs associated with individuals living in rural areas, and so, urban patients are more willing to substitute public for private care.

\section{Health Status}

Typically, health status is a variable that shifts the demand for medical services, decreasing demand for good health status and increasing it for bad health status [32]. This was precisely the pattern revealed by our model.

\section{Consultation}

From this group of variables, the most interesting variable is the 'not_enought_time' dummy variable. It is related to the quality dimension of care. Poor quality is usually associated to less utilization of care and to a higher willingness to substitute services. Contrarily, if the individual is unsatisfied with the consultation time, then he may increase demand to secure enough time with the GP. Nonetheless, we think that this potential effect is balanced by the other effect. In the frequent-users class this variable is not statistically significant perhaps due to the fact that frequent users tend to be patients who are having their diseases monitored. For them, consultation time is not as important as continuity of care.

Finally, regarding the interaction variables we did not find any statistically significant results.

\section{Conclusion}

The objective of this paper was to appraise the effect of time costs on the utilization of public GP consultations. We estimated the elasticity of utilization relative to the total time spent at the $\mathrm{HC}$ and relative to travel time. Moreover, we provided evidence regarding the impact of an appointment delay on the GP utilization. We have assumed that the monetary price to visit a GP is relatively unimportant when compared with the time costs, thus, these non-monetary costs may emerge as important determinants of utilization. The major conclusions that can be drawn from this research are the following: (1) the elasticity of demand relative to physical waiting is small or without statistical significance, and (2) users respond by decreasing utilization when they request care and do not see their request promptly satisfied without delay.

This study may have some policy implications for the Portuguese Health System. In Portugal there is a distortion on our gatekeeper system and as a consequence demand for emergency care is excessive, taking into account the general level of health status of the population. At the same time there is an evident lack of response from the primary health care system. An increase in the supply of medical care in health centres would have an impact on the appointment delay which, in turn, might reduce the demand for emergency care.

The results of our study would be more enlightening if we could have had the chance to include individuals' opportunity costs of time. The resulting estimates would not be as small as the ones we obtained [3]. 


\section{Acknowledgements}

The authors want to thank to the anonymous patients who answered to the Europe survey, and the Portuguese Institute for Quality in Health (IQS) that facilitated the implementation of the study. Our acknowledgements extend to Andrew Jones, João Santos Silva, Matthew Sutton, Pravin Trivedi, Pedro Pita Barros and to the participants in the 13th European Workshop on Econometrics and Health Economics held in Venafro, Italy. Two anonymous referees provided valuable and useful suggestions which improved the current paper as well as Teresa Lello who proofread the last version of the paper. The usual disclaimers apply.

\section{References}

1. Acton JP. Non-monetary factors in the demand for medical services: some empirical evidence. J Polit Econ 1975; 83: 595-614.

2. Phelps C, Newhouse J. Coinsurance, the price of time, and the demand for medical services. Rev Econ Stat 1974; 56(3): 334-342.

3. Coffey R. The effect of time price on the demand for medical care services. J Hum Resour 1983; 18(3): 407-424.

4. Janssen R. Time prices and the demand for GP services. Soc Sci Med 1992; 34(7): 725-733.

5. Dor A, Gertler P, Gaag VD. Non-price rationing and the choice of medical care providers in rural Cote D'Ivoire. J Health Econ 1987; 6: 291-304.

6. Cauley S. The time price of medical care. Rev Econ Stat 1987; 69(1): 59-66.

7. Lindsay CM, Feigenbaum B. Rationing by waiting lists. Am Econ Rev 1984; 74(3): 404-417.

8. Martin S, Smith PC. Rationing by waiting lists: an empirical investigation. J Public Econ 1999; 71: 141164.

9. Gravelle H, Dusheiko M, Sutton M. The demand for elective surgery in a public system: time and money prices in the UK National Health Service. J Health Econ 2002; 21: 423-449.

10. Blundell R, Windmeijer F. Identifying demand for health resources using waiting times information. Health Econ 2000; 9: 465-474.

11. Goddard A, Tavakoli M. Referral rates and waiting lists: some empirical evidence. Health Econ 1998; 7: 545-549.

12. Propper C. The disutility of time spent on NHS waiting lists. J Hum Resour 1995; 30(4): 677-701.

13. Cullis J, Jones P, Propper C. Waiting lists and medical treatment: analysis and policies. In Handbook of Health Economics, vol. 1, Culyer AJ, Newhouse JP (eds). Elsevier Science: Amsterdam, 2000; 1201-1249.

14. Oliveira M. Modelling demand and supply influences on utilization: a flow demand model to predict hospital utilization at small area level. Appl Econ 2004; 36: 2237-2251.

15. Barros PP, Olivella P. Waiting lists and patient selection. J Econ Manage Strategy 2005; 14(3): 623-646.

16. Becker G. A theory of the allocation of time. Econ J 1965; 75: 493-517.

17. Grossman M. On the concept of health capital and the demand for health. J Polit Econ 1972; 80(2); 223-255.

18. Cabral MV, Silva PA, Mendes H. Saúde e Doença em Portugal. Imprensa de Ciências Sociais: Lisboa, 2002.

19. Gertler P. Are user fees regressive? The welfare implications on health care financing in Peru. J Econometrics 1987; 36: 67-88.

20. Bolduc D, Lacroix G, Muller C. The choice of medical providers in rural Bénin: a comparison of discrete choice models. J Health Econ 1996; 15: 477-498.

21. Santos-Silva JMC. A note on the estimation of mixture models under endogenous sampling. Econometrics $J$ 2003; 36: 46-52.

22. Ferreira PL, Raposo V, Godinho P. A voz dos Utilizadores dos Centros de Saúde. IQS: Lisboa, 2005.

23. Bentes M, Dias CM, Sakellarides C, Bankauskaite V. Health care systems in transition: Portugal. WHO Regional Office for Europe on behalf of the European Observatory on Health Systems and Policies: Copenhagen, 2004.

24. Pinto CG, Oliveira M. The Portuguese Health Care System: current organization and perspectives of reform. In Como está a Economia Portuguesa? Centro de Investigaço sobre Economia Portuguesa (ed.). Europress; Lisboa, 2001; 161-194.

25. National Health Service Users Guide. http:// www.dgsaude.pt/ [03 May 2005].

26. Santana P. Geografia das desigualdades em saúde e no estado de saúde. In Livro de homenagem a Augusto Mantas. Barros PP, Simões J (eds). Associaço Portuguesa de Economia da Saúde: Lisboa, 1999; 179-235.

27. Pereira $\mathbf{J}$ et al. Health care reform and cost containment in Portugal. In Health Care and Cost Containment in European Union. Mossialos E, Le Grand J (eds). Ashgate: London, 1999.

28. Oliveira M. Bevan G. Measuring geographic inequalities in the Portuguese health care system: an estimation of hospital care needs. Health Policy 2003; 66(3): 277-292.

29. National Social Security web page. http://www.segsocial.pt/[05 May 2005].

30. Grol R, Wensing $\mathbf{M}$, Mainz $\mathbf{J}$ et al. Patients in Europe evaluate general practice care: an international comparison. Br J Gen Pract 2000; 50: 882 887.

31. Ferreira PL. A voz dos doentes: satisfaço com a medicina geral e familiar. In Instrumentos para a 
melhoria contínua da qualidade, Direcço-Geral da Saúde (eds). 1999.

32. Wagstaff A. The demand for health: some new empirical evidence. J Health Econ 1986; 5: 195-223.

33. Ramos P. Estudo sobre o Poder de Compra Concelhio. Instituto Nacional de Estatística: Lisboa, 2004.

34. Cameron C. Trivedi PK. Econometric models based on count data: comparisons and applications of some estimators and tests. $J$ Appl Econometrics 1986; 1: 29-53.

35. Deb P, Trivedi PK. The structure of demand for health care: latent class versus two-part models. $J$ Health Econ 2002; 21: 601-625.

36. Cameron AC, Trivedi PK. Regression analysis of count data. Econometric Society Monograph, vol. 30. Cambridge University Press: New York, 1998.

37. Grogger JT, Carson RT. Models for truncated counts. J Appl Econometrics 1991; 6: 225-238.

38. Brannas K, Rosenqvist G. Semiparametric estimation of heterogeneous count data models. Eur $J$ Oper Res 1994; 76: 247-259.

39. Gurmu S, Trivedi P. Overdispersion tests for truncated Poisson models. J Econmetrics 1992; 54: 347-370.

40. Wooldridge J. Econometric Analysis of Cross Section and Panel Data. MIT Press: Cambridge, MA, 2002.

41. Heckman JJ, Singer B. A method for minimizing the impact of distributional assumptions in econometric models for duration data. Econometrica 1984; 52: 271-320.

42. Deb P, Trivedi PK. Demand for medical care by the elderly: a finite mixture approach. J Appl Econometrics 1997; 12: 313-336.
43. Deb P, Holmes A. Estimates of use and cost of behavioural health care: a comparison of standard and finite mixture models. Health Econ 2000; 9: 475489.

44. Gerdtham UD, Trivedi P. Equity in Swedish health care reconsidered: new results based on the finite mixture model. Health Econ Lett 2001.

45. Atella V, Brindisi F, Deb P, Rosati F. Determinants of access to physician services in Italy: a latent class seemingly unrelated probit approach. Health Econ 2004; 13: 657-668.

46. Wedel M, Desarbo W, Bult J, Ramaswami V. A latent class Poisson regression model for heterogeneous count data. J Appl Econometrics 1993; 8: 397-411.

47. Sin C-Y, White H. Information criteria for selecting possibly misspecified parametric models. J Econometrics 1996; 71: 207-225.

48. Oehlert GW. A note on the Delta method. Am Stat 1992; 46(1): 27-29.

49. Irving L. Access to medical care: the Queen bridge experiment. Inquiry 1972; 9: 61-68.

50. Goldman F, Grossman M. The demand for paediatric care: an hedonic approach. $J$ Polit Econ 1978; 86(2): 259-280

51. Mwabu G, Ainsworth M, Nyamete A. Quality of medical care and choice of medical treatment in Kenya: an empirical analysis. J Hum Resour 1993; 28: $838-862$.

52. Akin JS, Griffin CG, Guilkey DK, Popkin BM. The demand for primary health care services in the Bicol region of the Philippines. Econ Dev Cultural Change 1986; 34(4): 755-783.

53. Mocan H, Tekin E. The demand for medical care in urban china. World Dev 2004; 32(2): 289-304. 